\title{
Sublimation Rate of TNT Microcrystals in Air
}

\author{
Arcady P. Gershanik ${ }^{\dagger}$ and Yehuda Zeiri ${ }^{*, \dagger}$, \\ Biomedical Engineering, Ben Gurion University of the Negev, Beer-Sheva 84105, Israel, and Division of \\ Chemistry, NRCN, Beer-Sheva P.O. Box 9001, 84190, Israel
}

Received: June 5, 2010; Revised Manuscript Received: October 15, 2010

\begin{abstract}
Reliable measurements of the sublimation rate of nonuniform layers of trinitrotoluene (TNT) microcrystals were carried out using a quartz crystal microbalance $(\mathrm{QCM})$. The sample layer was prepared by precipitation of TNT from a well-defined volume of its solution in acetonitrile. The TNT solution was placed on the QCM electrode surface to form the precipitated layer of TNT microcrystals. It is shown that the kinetics of small TNT particles sublimation is controlled by the molecular diffusion in air. The sublimation process is well described by simple diffusion expressions that are discussed in the literature for both individual hemisphericalshaped microcrystals and disk-shaped layers. Expressions describing particle size evolution in time were derived based on this diffusion model. It is shown that the expressions developed can be used to simulate particle sublimation in a wide size range, including very small sub-micrometer particles.
\end{abstract}

\section{Introduction}

For over a decade, large amounts of governmental resources have been channeled into the war against terrorism by most of the countries all over the globe. Large efforts are devoted to maintain secure public transportation, in particular the air traffic. The meaning is to do all that one can to eliminate the possibility of smuggling explosives into airports and airplanes. The problem is that the vapor pressure of most solid explosives, both military and improvised, is too low to detect their presence using methods that examine their gas phase existence. Thus, the most promising way to detect the explosive is to sample micrometer (or less) size explosive particles that may be found on the belongings or clothing of people that were exposed to explosive in manufacturing or assembling laboratories. The sampling in most cases is performed by a swipe of the examined surface using a swipe material and detecting the existence of explosives on the swipe material by an ion mobility spectrometer (IMS).

The fate of explosive particles on various substrates related to terrorist or criminal acts is important if one desires to optimize the sampling of explosive particles. As in an open system, explosive and all kind of particles are expected to evaporate and disappear; hence, knowledge of their lifetime in various environmental conditions is important. For most solid explosives, the vapor pressure is rather low, and its evaporation in air may occur during a relatively long time scale. A particle's lifetime is of interest since it determines the possibility to sample and detect particles in various scenarios related to terrorist and criminal activities. Moreover, knowledge of the sublimation rate dependence on the particle dimension would give the possibility of restoring their initial distribution at given environmental conditions.

The sublimation process is based on two fundamental events: the first is desorption of molecules from the solid, and the second is the motion of the gas phase molecules into the surrounding and away from their original source. This description is valid only if the desorption process is a reversible one, namely, the

\footnotetext{
* To whom correspondence should be addressed. E-mail: yehuda@bgu.ac.il.

$\uparrow$ Ben Gurion University of the Negev.

* NRCN.
}

desorbing molecules do not alter chemically in a non-reversible manner (i.e., they do not decompose).

If the sublimation source consists of small particles, additional considerations have to be taken into account. Among these are the role of air flow on the diffusion process and the enlarged deviation from equilibrium of the maximal vapor concentration in the vicinity of the solid.

As will be discussed below, the estimate of sublimation rate based on diffusion equations requires knowledge of the diffusion coefficients of the sample molecules in air and the values of the sample saturation vapor pressures. As mentioned, the vapor pressure values of most explosives are quite low. As a result, considerable discrepancies of up to a factor of 2-3 and sometimes more are found in the published experimental data. ${ }^{1-6}$ The experimental reports of the TNT diffusion coefficient in air also differ. The variation between the reported values for TNT diffusion in air $^{7-9}$ spans up to $30 \%$. The theoretical estimations of the diffusion coefficient by several different methods differ by a factor of $3 .{ }^{10}$

Contrary to the nature of the sublimation process discussed above, a recent publication, related to TNT sublimation, suggested that influence of the diffusion-in-air retardation on the overall rate is negligible. ${ }^{11}$ It will be demonstrated that the description of TNT sublimation kinetics as a diffusion process agrees well also with the experimental results of ref 11 .

The quartz crystal microbalance (QCM) is a suitable instrument to measure sublimation rates due to its very high sensitivity to minute mass changes, on the order of sub-nanograms. However, QCM can be used only if some requirements related to the physical, chemical, and mechanical properties of the sample are met. In general, the substance under investigation should have good adhesion to the QCM surface, should be homogeneously spread, and should be sufficiently rigid. ${ }^{12,13}$ QCM may lose its high sensitivity in the absence of good adhesion or undergo additional frequency shifts in the case of soft sample due to dissipation of oscillation energy. ${ }^{14,15}$ Large errors in the measurement may occur in the case of thick nonrigid layers due to the acoustic wave absorption by the soft load. 
In this work we used a quartz crystal microbalance (QCM) to study the sublimation rate of trinitrotoluene (TNT) microcrystals. The microcrystal layer was directly precipitated from solution onto the QCM electrode surface. The main goal in this study is to understand the mechanism governing the TNT sublimation process and to develop an appropriate model to quantify it.

\section{QCM Measurements}

2.1. General Consideration. It is known from the literature ${ }^{13}$ that the frequency shift of the QCM that is caused by a nonuniform load is equal to the sum of the partial shifts resulting from the mass distributed over the QCM surface. A different sensitivity at different points of the QCM electrode should be taken to account, that is,

$$
\Delta f=\int_{0}^{2 \pi} \int_{0}^{r_{\mathrm{q}}} \xi(r, \theta) \rho_{\mathrm{s}}(r, \theta r, \theta) \mathrm{d} r \mathrm{~d} \theta
$$

where $\Delta f$ is the measured frequency shift relative to the fundamental, $r$ and $\theta$ are the polar coordinates of different points along the crystal surface, $r_{\mathrm{q}}$ is the electrode radius, $\rho_{\mathrm{s}}$ is the density of the sample mass, and $\xi$ is a local sensitivity function that is independent of the magnitude of the mass in the approximation of small loads.

Extracting the average value of the sensitivity $\bar{\xi}$ and of the mean sample density along the QCM surface $\bar{\rho}_{\text {s }}$ from the integral (1) one obtains

$$
\Delta f=\bar{\xi} \overline{\rho_{\mathrm{s}}} \int_{0}^{2 \pi} \int_{0}^{r_{\mathrm{q}}} F(r, \theta) \mathrm{d} r \mathrm{~d} \theta=-\frac{\bar{\xi} m}{A} B
$$

with

$$
B=\int_{0}^{2 \pi} \int_{0}^{r_{\mathrm{q}}} F(r, \theta) \mathrm{d} r \mathrm{~d} \theta
$$

where $A$ is the QCM electrode surface area and $F(r, \theta)$ represents the reduced distribution function of the load that is independent of the absolute value of mass.

When the distribution is homogeneous (i.e., $B=1$ ), eq 2 is essentially identical to Sauerbrey's equation. ${ }^{12}$ In the inhomogeneous mass distribution case, the $B$ value may change from one sample to another but can be assumed to be almost constant during the initial stages of the sample sublimation, before the reduced mass distribution function changed appreciably. In such situation, eq 2 can be rewritten in the following form to describe the sublimation process

$$
\begin{gathered}
\Delta(\Delta f)=-\frac{\overline{\xi \Delta} m}{A} B \\
\frac{\Delta(\Delta f)}{\Delta f 0}=-\frac{\Delta m}{m_{0}}
\end{gathered}
$$

where $\Delta f_{0}$ is initial frequency shift after deposition of the initial mass $m_{0}$ on the electrode surface.

Thus, measurement of the resonance frequency shift following sample loading allows one to detect the changes in the sample due to evaporation. This type of measurement can yield satisfactory means to study the sublimation rate of various materials of interest.
The accuracy of this approach to measure lifetime of particles has been examined here using several means. First, the reproducibility of the measurements was examined following consecutive loading of the QCM surface by samples. The good reproducibility obtained in the sublimation rate measurements confirms our assumption concerning the strength of the sample adhesion to the QCM electrode surface. However, the data analysis can be complicated by a strong dependence of the measured evaporation rates on the detailed structure of the sample microcrystals layer. Hence, a verification of the sublimation rate measurement approach was obtained by comparison between the data obtained by QCM with an independent measurement of the evaporation rate using an analytic balance (ViBRA).

2.2. Experimental Procedure. TNT Solution. Military standard 2,4,6-trinitritoluene solution in acetonitrile with TNT concentration of $10 \mathrm{~g} / \mathrm{L}$ and acetonitrile purity of $99.9 \%$ was received from Rafael Ltd. This TNT concentration was found to be too high to obtain reproducible microcrystal layers. Good reproducibility of the sample layer has been achieved with a more diluted TNT solution ( $2 \mathrm{~g} / \mathrm{L})$. The initial sample layer was created by TNT microcrystals precipitation during the evaporation of the solvent from $10 \mu \mathrm{L}$ TNT solution placed on the QCM electrode. The solvent was allowed to evaporate at room temperature. The microcrystalline layer formed during solvent evaporation was documented by an optical microscope at various magnifications.

Equipment and Procedure. The quartz microbalance used is model QCM100 by Stanford Research Systems, Inc. The ATcut disk-shaped quartz crystal of 1 in. diameter was covered in the central area ( 0.5 in. diameter) by a thin gold layer. According to the manufacturer data, the crystal holder withstands temperature up to $85^{\circ} \mathrm{C}$. However, the preamplifier attached by a BNC connection to the crystal holder may be exposed only to temperatures up to $40{ }^{\circ} \mathrm{C}$. To eliminate this restriction, in all experiments reported here the preamplifier was separated from the crystal holder by a $10 \mathrm{in.} \mathrm{long} \mathrm{cable.} \mathrm{The} \mathrm{temperature} \mathrm{control}$ was achieved by placing the crystal holder with the sample on the crystal surface in an oven with polished stainless steel walls with ventilation located at the top of the oven. Before placing the sample in the oven it was heated to the desired temperature. The sublimation experiments reported below were carried out at constant temperature with fluctuations less than $\pm 0.2{ }^{\circ} \mathrm{C}$.

The quartz crystal holder was usually placed on the bottom of the oven at a distance $5-6 \mathrm{~cm}$ from one of the walls with the sample facing the wall. The position of the crystal was chosen to be as far as possible from the ventilator. After placing the crystal holder in the oven at the desired temperature, the crystal and the sample reached the desired temperature within approximately $5 \mathrm{~min}$. The beginning of the data acquisition was delayed by this period.

To eliminate the accumulation of the TNT vapors in the volume above the sample during the sublimation process, the oven was pumped out continuously using a small membrane pump (pumping rate of $18 \mathrm{~L} / \mathrm{min}$ ).

As mentioned above, to verify the results obtained using the QCM experiments, we performed a similar experiment using an analytical balance. This experiment was carried out in the following way. A layer of TNT was precipitated on both sides of a large, thin plastic disk (diameter of $100 \mathrm{~mm}$ ) using the 2 $\mathrm{g} / \mathrm{L}$ TNT solution in acetonitrile. The total amount of TNT precipitated on the sample plate was $15 \mathrm{mg}$. The sample was placed in the oven on the disk ledge, with both faces available for evaporation, at temperature of $T=30^{\circ} \mathrm{C}$. The change in 


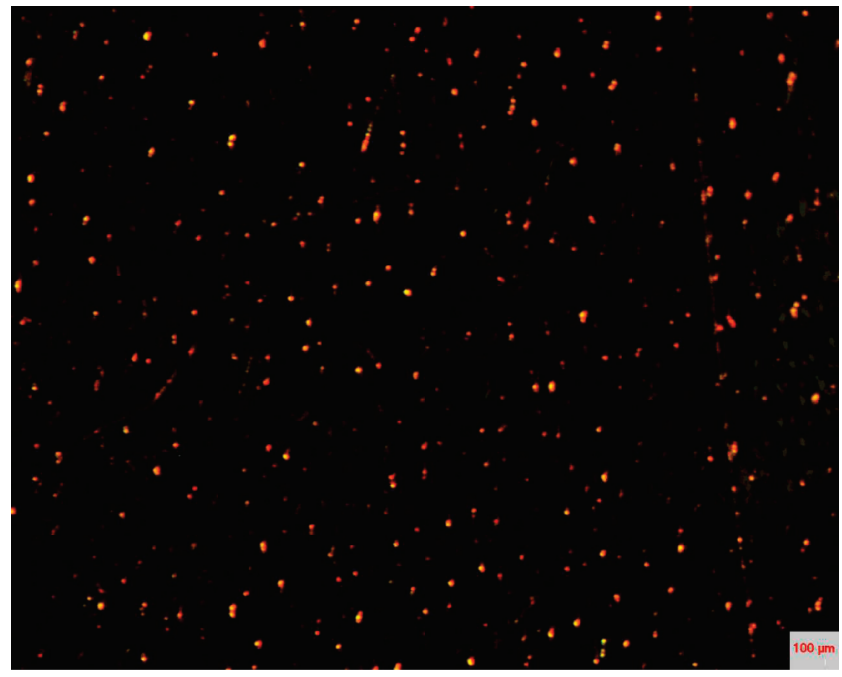

Figure 1. Typical optical microscope image of the TNT microcrystal layer near the central part of QCM electrode following precipitation of the microcrystals from a $2 \mathrm{~g} / \mathrm{L}$ solution.

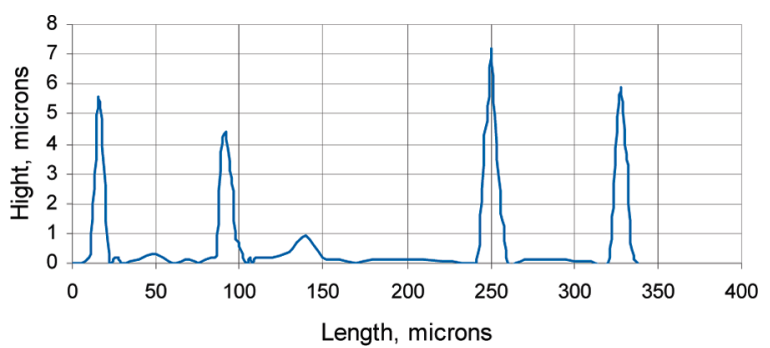

Figure 2. Typical spectrum of the TNT micro crystal layer near the central part of QCM electrode as obtained by the Dektak profiler measurements.

the disk weight was measured every hour to obtain a direct measure of the fraction evaporated from the TNT layer.

2.3. Results. A typical optical microscope image of the initial TNT layer near the central part of the QCM electrode is shown in Figure 1. One can see clearly that the initial morphology of the TNT layer is composed of well-separated TNT microcrystals. Simple image analysis of many images such as the one shown in Figure 1, at different magnifications, allowed us to obtain an estimate of the average microcrystal diameter and the average distance between neighboring microcrystals. In the image analysis the particle area is measured and converted to diameter approximating the shape by a circle. The average diameter of these microcrystals was found to be in the range of $10-12 \mu \mathrm{m}$. The average distance between neighboring particles was found to be of the order of $100 \mu \mathrm{m}$, much larger than the particles diameters. The corresponding standard deviations of these two quantities are: $20 \%$ and $36 \%$ respectively.

To obtain information about to the height of the TNT microcrystals, stylus surface profiler (Dektak 8) was used. This system offers flexible stylus forces normal to the substrate of $0.03 \mathrm{mg}$ with height resolution of $0.75 \mathrm{~nm}$. The profiler software allows simultaneous observation through the microscope of the particle together with the stylus. This feature helps to ensure that the stylus do not move the particle. The typical height variation along an arbitrary line across the sample is shown in Figure 2. The average height of the TNT microcrystals obtained is $5.9 \mu \mathrm{m}$ with a standard deviation of $0.9 \mu \mathrm{m}$.

Ideally, one would like to have a layer composed of identical particles that would enable an easier understanding of the system behavior. Unfortunately, this is impossible for layers that are

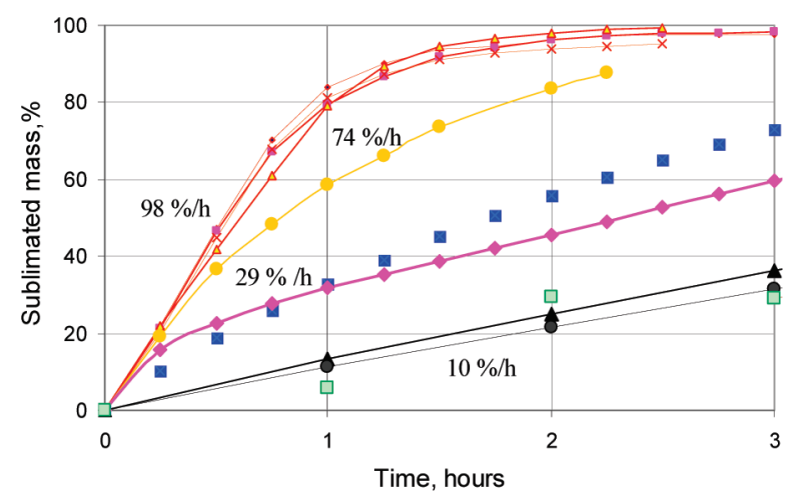

Figure 3. Sublimation rate of the precipitated TNT layer measured by QCM at several temperatures: red lines correspond to $50{ }^{\circ} \mathrm{C}$, yellow corresponds to $45{ }^{\circ} \mathrm{C}$, magenta corresponds to $40{ }^{\circ} \mathrm{C}$, and black corresponds to $30{ }^{\circ} \mathrm{C}$. Blue rectangles correspond to data obtained in an experiment at $40^{\circ} \mathrm{C}$ with the sample positioned as close as possible to the ventilator in the oven. The green points correspond to data obtained in the experiment using the analytical balance to follow weight changes. Numbers adjacent to the curves indicate the average value of the initial sublimation rate.

formed by precipitation processes. Moreover, a full statistical treatment using the particle geometry distribution cannot be carried out due to the unknown dependence of the QCM sensitivity on the location of the various particles. Analysis of the optical microscope images together with the profiler data clearly shows that the precipitated layer consists, to a very good approximation, of separated particles whose average radius and height is approximately $6 \mu \mathrm{m}$. It is clear that the actual shape of the particles exhibits wide variations due to the nature of the process used to form the layer. However, in the following a simple model to describe the sublimation rate of the microparticles will be described. To keep this model simple we shall approximate the shape of the individual particles by hemispheres with radius of $6 \mu \mathrm{m}$. It will be demonstrated that this approximation allows one to obtain reasonable agreement between the experimental data and the model predictions. Some of the uncertainties associated with the various approximations used will be further discussed below.

The mass fraction change during the sublimation process as measured by the QCM is shown in Figure 3 for four different temperatures. Two points should be noted: (i) Measurements in which the analytical balance was used to monitor weight change at $30{ }^{\circ} \mathrm{C}$ confirm the reliability of the data obtained by the QCM. This substantiates the validity of evaporation rate measurements using the QCM. (ii) The air flow induced by the oven ventilator does not lead to significant changes in the measured evaporation rate. The reproducibility of the QCM measurements at any given temperature is very good, achieving $\pm 7 \%$ of the measured value at highest temperature $50{ }^{\circ} \mathrm{C}$ and $\pm 15 \%$ at the lowest temperature $30{ }^{\circ} \mathrm{C}$. These results suggest that the approach used for sample preparation yield statistically similar initial sample layer morphologies. The blue and magenta curves in Figure 3 corresponds to two experiments carried out at $40{ }^{\circ} \mathrm{C}$, first with sample placed facing the oven wall and far from the ventilator (magenta) and the other with sample placed as close as possible to the ventilator in the oven (blue). The results of these two experiments show deviations of approximately $20 \%$. This deviation is somewhat greater than in the other measurements performed at 30 and $50{ }^{\circ} \mathrm{C}$. However, this deviation is mainly related to the thermo-mechanical action of the air flow on the QCM.

The initial slope of the various curves in Figure 3 is related to the evaporation rate of the sample. The magnitude of the 


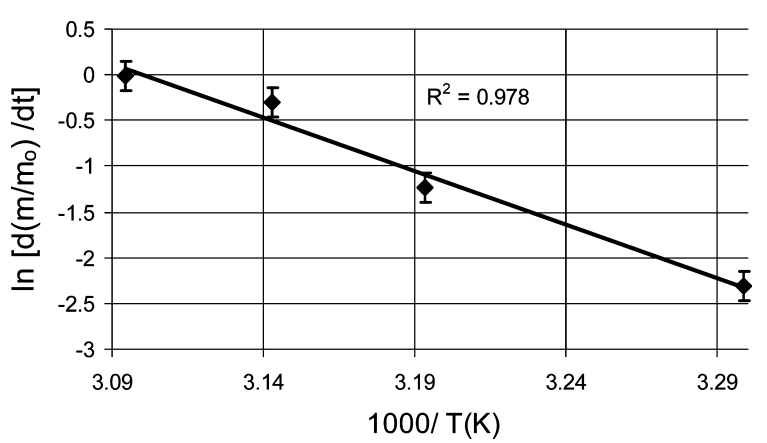

Figure 4. Dependence of the initial sublimation rate on inverse temperature.

initial slopes, marked on Figure 3, was obtained by a linear fit of the sublimated mass curves in their linear regions.

If one assumes an Arrhenius form for the temperature dependence of evaporation rates, then the activation energy of the process can be obtained from the slope of the linear dependence between the natural logarithm of the sublimation rate and the inverse absolute temperature. Figure 4 shows the experimental points together with the best fit of linear variation line. The activation energy, related to the slope, associated with the TNT sublimation process is $97 \pm 7 \mathrm{~kJ} / \mathrm{mol}$. This value is very close to the heat of sublimation $99 \mathrm{~kJ} / \mathrm{mol}$ obtained in ref 1 but is considerably smaller than the largest among the reported values $^{1-6}$ of $141 \mathrm{~kJ} / \mathrm{mol}^{6}$

\section{Discussion}

The sublimation process consists at least of two sequential basic events: a stage of the molecules escaping (desorption) from the solid and the motion of these molecules away from the vicinity of the solid. These two stages are considered in more details below.

3.1. Desorption Process. The rate of molecules escaping from solid is easily calculated from the value of equilibrium vapor pressure using the well-known Langmuir-Knudsen approach for vapor pressure measurements ${ }^{16,17}$ that gives

$$
-\frac{\mathrm{d} m}{\mathrm{~d} t}=g \frac{A_{0} P_{\mathrm{sat}}}{\sqrt{2 \pi M k B T}}
$$

where $\mathrm{d} m / \mathrm{d} t$ is the rate of sample mass change due to the sublimation, $A_{0}$ denotes the sample surface area, $P_{\text {sat }}$ is the saturation vapor pressure of the sample material, $T$ is the temperature, $k_{\mathrm{B}}$ is Boltzman's constant, $M$ is the molecular mass, and $g$ represents the sticking coefficient that is usually considered as close to unity.

To the best of our knowledge, the experimental values of sticking coefficient for TNT were not reported in literature. Moreover, all the vapor pressure measurements ${ }^{1-3}$ that were carried out by the Langmuir approach assumed that $g=1$. In other words, the reported vapor pressure data are really a measure of the product $g P_{\text {sat }}$ rather than of $P_{\text {sat }}$ by itself. Therefore, using the vapor pressure data obtained in the Langmuir-Knudsen type experiments allows replacing of the product $g P_{\text {sat }}$ by the experimentally obtained effective $P_{\text {sat }}$.

For a hemispherical particle of radius $r_{0}$ and initial mass $m_{0}$, one can rewrite eq 6 in the following form

$$
-\mathrm{d}\left(m / m_{0}\right)=\frac{3 P_{\text {sat }}}{r_{0} \rho} \sqrt{\frac{M}{2 \pi k_{\mathrm{B}} T}}
$$

where $\rho$ represents the sample density.
Equation 7 relates to a hemispherical particle, that is, the shape that possess the smallest ratio of surface area to volume (or mass). Consequently, the calculations can be regarded as a lower limit for the evaporation rate since any deviation from the hemispherical particle shape corresponds to larger surface volume ratio. To estimate the minimal TNT mass loss rate according to eq 7 at $30{ }^{\circ} \mathrm{C}$ we used the largest and the smallest vapor pressures reported in the literature..$^{1-6}$ The largest reported value of TNT vapor pressure is $2.38 \times 10^{-3} \mathrm{~Pa}^{1}$, which yields an evaporation rate of $0.27 \% / \mathrm{s}$ while the smallest value, $P_{\text {sat }}=$ $1.05 \times 10^{-3} \mathrm{~Pa}$, reported by Oxley et al. ${ }^{4}$ yields a sublimation rate of $0.12 \% / \mathrm{s}$. The corresponding measured rate in the QCM experiments was $2.8 \times 10^{-3} \% / \mathrm{s}$. This discrepancy of about 2 orders of magnitude suggests that most of the desorbing TNT molecules are readsorbed by the solid after collisions with the surrounding air molecules. As a result, in the near vicinity of the particle surface an equilibrium concentration of the sample molecules is maintained. Thus, to describe properly the evaporation process one needs to take into account the diffusion of the sample molecules in the surrounding air using the boundary condition described above. This second stage of the sublimation process is discussed below.

3.2. Diffusion in Air. Let us consider the diffusive motion of sample molecules in the gas phase after their desorption from the TNT microcrystal surface. It is assumed that molecular diffusion is the main process that governs the motion of the sample molecules while they leave the particle vicinity. In other words, various air flows leading to convective or forced motion are neglected. This is justified due to the nature of the flow near small particles as discovered and measured experimentally by Langmuir. ${ }^{18}$ Below we will present a quantitative estimation of the particle dimension that satisfies these conditions.

Let us consider the quasi-stationary steady state diffusion process. The solution of the Fick's first law of molecular diffusion for a hemispherical particle that is placed on a substrate has the form ${ }^{19}$

$$
C(r)=C_{\max } \frac{r_{0}}{r}
$$

where $C_{\max }$ represents the concentration of the sample molecules in the gas phase next to the particle surface, $r_{0}$ is the particle radius, and $r$ is the independent variable in the range $r_{0}$ to infinity.

According to eq 8 , the gas phase molecular concentration $C(r)$ decreases along the radial direction as $1 / r$. At small distances from the particle, of order of ten times the particle radii, the concentration $C(r)$ becomes negligibly small relative to $C_{\max }$. In other words, the main resistance to the diffusion process is most effective in the region next to the particle surface. If this region is within the limits of the boundary layer (a motionless gas layer whose width can vary in the range of few micrometers up to few millimeters or more depending on the conditions in the system) then, to a very good approximation, air flow is not expected to alter the concentration of TNT molecules and their transport. The small volume of this region requires only very small amount of sublimated substance to achieve steady-state gas molecules concentration. This is especially true in case of TNT and similar substances with low vapor pressure. For example, the density of the solid TNT is approximately $10^{10}$ times greater than the density of its saturate vapor at room temperature.

Remembering that for ideal gas 


$$
C_{\max }=\frac{P_{\text {sat }}}{k_{\mathrm{B}} T}
$$

Substitution of eqs 8 and 9 into Fick's first law expression, one obtains for the sublimation rate the following expression for hemispherical particle

$$
-\frac{\mathrm{d} m}{\mathrm{~d} t}=\frac{2 \pi D P_{\mathrm{sat}} r_{0}}{k_{\mathrm{B}} T}
$$

where $D$ denotes the TNT diffusion coefficient in air. For the fractional mass change one obtains

$$
-\mathrm{d}(m / m 0) / \mathrm{d} t=\frac{3 D P_{\mathrm{sat}} M}{r_{0}^{2} \rho k_{\mathrm{B}} T}
$$

Equation 11 describes the fractional mass change for hemispherical particle was applied to model the sublimation rate of TNT particles investigated here. The coefficient 3 in eq 11 can be regarded as a form-factor related to the hemispherical form of the particles. This form factor is an approximation of the actual mean statistical form-factor of the precipitated TNT layer whose exact value is unknown. To the best of our knowledge, analytical forms of the steady state solutions of Fick's equation for the vapor diffusion from a small source into open space are available in literature only for hemispherical- and disk-shaped sources. ${ }^{20}$ Therefore, the form factor for an arbitrary shaped particle is not available. According to Gray et al., ${ }^{20}$ the corresponding sublimation rate equation for a disk of radius $r_{\mathrm{d}}$ is given by the following expression

$$
-\mathrm{d} m / \mathrm{d} t=\frac{4 D P_{\mathrm{sat}} r_{\mathrm{d}}}{k_{\mathrm{B}} T}
$$

Comparison with eq 10 for the mass loss by a hemispherical particle source shows that the form factor for a hemisphere is $\pi / 2$ times greater than that for a disk-shaped source. This difference can serve as an approximate measure of the formfactor variation for different shape particles.

Knowledge of the saturated vapor pressures and the magnitude of the diffusion coefficients for TNT are required. However, saturated vapor pressures reported in the literature show (see discussion above) large discrepancies, up to factor of $2-3$. $^{1-6}$ For TNT diffusion coefficient the reported values show discrepancy of about $30 \% .^{7-9}$ Therefore, for the analysis described below we use an average value of the diffusion coefficients reported in the litrature, ${ }^{7-9} D=5.59 \times 10^{-6} \mathrm{~m}^{2} / \mathrm{s}$, and we neglect its slight temperature dependence in the narrow temperature range studied here.

The vapor pressure data reported in the litrature ${ }^{1-6}$ are shown in Figure 5 for the temperature range of $25-50{ }^{\circ} \mathrm{C}$. It is quite clear that this data can be subdivided into two main groups (refs $1-3$ and refs $4-6$ ) with considerably different slopes. Note that all vapor pressure values seem to converge to the same value near $50{ }^{\circ} \mathrm{C}$. The difference in the slopes of these two groups reflects the corresponding difference in the heat of sublimation values. The source of this difference is unclear to us. Our estimate of the heat of sublimation to be $97 \mathrm{~kJ} / \mathrm{mol}$ (see Figure 4 ) is close to the data of the first group (the low slope group). ${ }^{1-3}$

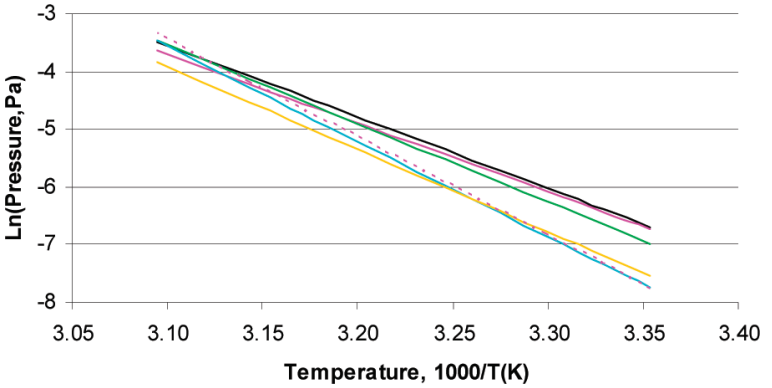

Figure 5. TNT vapor pressure data in the temperature range $25-50$ ${ }^{\circ} \mathrm{C}$ : black line presents data of Linchitz et al.; ${ }^{1}$ red line, of Pella; ${ }^{2}$ green line, of Cundall et al. ${ }^{3}$ blue line, of Oxley et al.; ${ }^{4}$ yellow line, of

\begin{tabular}{|c|c|c|c|c|c|c|}
\hline \multirow{2}{*}{$\frac{\mathrm{A}}{t,{ }^{\circ} \mathrm{C}}$} & \multirow{2}{*}{$\begin{array}{c}\text { measured, } \\
1 / \mathrm{h}\end{array}$} & \multicolumn{4}{|c|}{ calculated using vapor pressure of: } & \multirow{2}{*}{$\frac{\text { ratio }}{\text { calc/meas. }}$} \\
\hline & & Lenchitz $^{1}$ & 1 Pella $^{2}$ & Cundall $^{3}$ & $\overline{\text { average }}$ & \\
\hline 30 & 0.1 & 0.22 & 0.21 & 0.18 & 0.20 & 2.02 \\
\hline 40 & 0.29 & 0.78 & 0.72 & 0.7 & 0.74 & 2.55 \\
\hline 45 & 0.74 & 1.44 & 1.29 & 1.40 & 1.38 & 1.86 \\
\hline 50 & 0.98 & 2.59 & 2.26 & 2.67 & 2.51 & 2.56 \\
\hline B & \multirow{2}{*}{$\begin{array}{c}\text { measured, } \\
1 / \mathrm{h}\end{array}$} & \multicolumn{4}{|c|}{ calculated using vapor pressure of: } & ratio \\
\hline$t,{ }^{\circ} \mathrm{C}$ & & Oxley $^{4}$ & Edwards ${ }^{5}$ & Leggett ${ }^{6}$ & average & calc/meas \\
\hline 30 & 0.10 & 0.10 & 0.11 & 0.10 & 0.10 & 1.02 \\
\hline 40 & 0.29 & 0.52 & 0.53 & 0.68 & 0.58 & 1.99 \\
\hline 45 & 0.74 & 1.18 & 1.08 & 1.60 & 1.29 & 1.74 \\
\hline 50 & 0.98 & 2.59 & 2.15 & 3.66 & 2.80 & 2.86 \\
\hline
\end{tabular}
Edwards; ${ }^{5}$ and magenta line, of Leggett. ${ }^{6}$

TABLE 1: Comparison of Calculated and Experimental Sublimation Rates at Different Temperatures ${ }^{a}$

${ }^{a}$ Part A presents the calculated results using the measured vapor pressures that corresponds to lower heats of evaporation (first group in Figure 5), whereas part B presents the calculated values using the vapor pressures that corresponds to higher heats of evaporation (second group in Figure 5).

The calculated sublimation rates, using all the reported vapor pressure, are summarized in Table 1, parts A and B, together with the measured rates as obtained in the QCM experiments. The calculated rates, using all the $P_{\text {sat }}$ values of refs $1-6$ yield a sublimation rate whose magnitude is approximately twice the measured rates with only small dependences on temperature. Taking into account the uncertainties of the TNT microcrystals size and shape distributions, the agreement between the measured and calculated values is very satisfactory.

Sublimation rates of the TNT samples with a much better definition of the sample initial geometry is presented in the paper of $\mathrm{Mu}$ et al. ${ }^{11}$ In their study of the interaction of TNT layers with several substrates, the authors measured the sublimation rate in air of a thin TNT disk-shaped layer formed by vapor deposition on the QCM electrode surface. The thickness of the vapor deposited disk in this study was $12 \mu \mathrm{m}$ with a diameter of $6 \mathrm{~mm}$.

The description of the sublimation process given here is different than that given by $\mathrm{Mu}$ et al. ${ }^{11}$ despite the fact that the method of measurement is very similar. In the study of ref 11 , the authors neglected the retardation effect of the air on the TNT sublimation from a disk-shaped sample. In the following we shall reanalyze the data obtained by $\mathrm{Mu}$ et al. ${ }^{11}$ using the simple diffusion-based description discussed above.

A comparison between the experimental data presented in ref 11 and the calculated values obtained by eq 12 for a diskshaped sample is shown in Table 2, parts A and B, for the first and second groups of reported vapor pressures discussed above. The value of the diffusion coefficient used was taken as an 
TABLE 2: Comparison of the Experimental Data of Mu et al. ${ }^{11}$ with Calculated Rates According to Equation $12^{a}$

\begin{tabular}{|c|c|c|c|c|c|c|}
\hline \multirow{2}{*}{$\frac{\mathrm{A}}{t,{ }^{\circ} \mathrm{C}}$} & \multirow[b]{2}{*}{ measured, ng/s } & \multicolumn{4}{|c|}{ calculated using vapor pressure of: } & \multirow{2}{*}{$\frac{\text { ratio }}{\text { calc/meas }}$} \\
\hline & & Lenchitz $^{1}$ & Pella $^{2}$ & Cundall $^{3}$ & average & \\
\hline 25 & $7.54 \times 10^{-3}$ & $7.19 \times 10^{-3}$ & $7.34 \times 10^{-3}$ & $5.63 \times 10^{-3}$ & $6.72 \times 10^{-3}$ & 0.89 \\
\hline 30 & $1.47 \times 10^{-2}$ & $1.44 \times 10^{-2}$ & $1.40 \times 10^{-2}$ & $1.17 \times 10^{-2}$ & $1.34 \times 10^{-2}$ & 0.91 \\
\hline 40 & $5.56 \times 10^{-2}$ & $5.17 \times 10^{-2}$ & $4.74 \times 10^{-2}$ & $4.75 \times 10^{-2}$ & $4.89 \times 10^{-2}$ & 0.88 \\
\hline $\mathrm{B}$ & & \multicolumn{4}{|c|}{ calculated using vapor pressure of: } & ratio \\
\hline$t,{ }^{\circ} \mathrm{C}$ & measured, ng/s & $\mathrm{Oxley}^{4}$ & Edwards $^{5}$ & Leggett $^{6}$ & average & calc/meas \\
\hline 25 & $7.54 \times 10^{-3}$ & $2.58 \times 10^{-3}$ & $3.24 \times 10^{-3}$ & $2.69 \times 10^{-3}$ & $2.84 \times 10^{-3}$ & 0.38 \\
\hline 30 & $1.47 \times 10^{-2}$ & $6.35 \times 10^{-3}$ & $7.00 \times 10^{-3}$ & $6.77 \times 10^{-3}$ & $6.71 \times 10^{-2}$ & 0.46 \\
\hline 40 & $5.56 \times 10^{-2}$ & $3.46 \times 10^{-2}$ & $3.04 \times 10^{-2}$ & $3.92 \times 10^{-2}$ & $3.48 \times 10^{-2}$ & 0.63 \\
\hline
\end{tabular}

${ }^{a}$ Part A presents the calculated results using the measured vapor pressures that corresponds to lower heats of evaporation (first group in Figure 5), whereas part B presents the calculated values using the vapor pressures that corresponds to higher heats of evaporation (second group in Figure 5).

average of the reported data in the litrature. ${ }^{7-9}$ The agreement between the calculated values and the measurements is quite good, with differences in the range of $9 \%$ up to $60 \%$ only. It should be noted that using the vapor pressures that correspond to an evaporation heat of $99 \mathrm{KJ} / \mathrm{mol}$ yields sublimation rates in very good agreement with the measured values, whereas using the second group of vapor pressures results in a slightly poorer agreement. These findings suggests that the roughly 2-fold deviation between the calculated and the measured values is observed in Table 1 are mainly due to the inaccuracy introduced by the assumption of identical hemispherical particles. In addition, the rather good agreement between the experimental data and the calculated rates in Table 2 suggest that the sublimation rate of TNT for the disk-shaped samples ${ }^{11}$ were also limited by the diffusion process. It should be noted that, according to eq 12 , the sublimation rate is proportional linearly to the sample dimension. This is in contradiction with the authors DIDP model ${ }^{21}$ where the sublimation rate in air was assumed to be proportional to the sample area.

Finally, the conclusion about the leading role of diffusion retardation impact on the sublimation process allows describing the time variation of the TNT particle dimensions due to the sublimation process. Let us turn back to the steady state diffusion expression, eq 10. Assuming the validity of the same steady state quasi-stationary approximation, it is possible to replace the constant radius $r_{0}$ of the particle by its time dependent magnitude, $r_{0}(t)$. This approximation is based on the very large difference between the densities of the solid and the vapor phases. For example, if a volume of 10 particle radii surrounding the particle is filled at $30{ }^{\circ} \mathrm{C}$ by the saturated vapor pressure of TNT, the amount of TNT molecules required to obtain this concentration leads to a contraction of a hemispherical particle by $5 \times 10^{-9}$ of its radius. The evaporation rate of the particle will yield $P_{\text {sat }}$ in this volume in approximately $2 \mu \mathrm{s}$.

Integration of eq 10 over time yields the variation of the particle size as a function of time. After substituting in eq 10 instead of mass, $\mathrm{m}$, the corresponding time dependent value the variation of particle radius can be obtained in the form

$$
r_{0}(t)=\sqrt{r_{0}^{2}(t=0)-\frac{2 D P_{\mathrm{sat}} M}{\rho k_{\mathrm{B}} T} t}
$$

Typical variation of TNT particle size at $T=30{ }^{\circ} \mathrm{C}$ according to eq 13 is shown in Figure 6. It is important to point out that eq 13 cannot be justified for very small particles whose size is comparable with the mean free path of the sample molecules

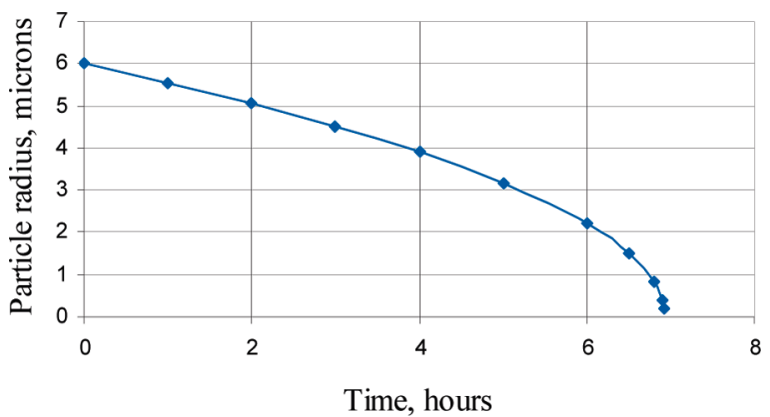

Figure 6. Time dependence of particle radius for a hemispherical TNT particle with initial radius of $6 \mu \mathrm{m}$ at $30^{\circ} \mathrm{C}$.

in air, of the order of $0.1 \mu \mathrm{m}$. There are a few reasons that eq 13 is incorrect in such situation, the first, and most important, is that the applicability of eq 8 for the concentration distribution breaks down for very small particles. Equation 8 was derived by rigorous integration of Fick's differential equation in the interval starting at the hemisphere boundary, $r_{0}$, and extends to large arbitrary $r$ values.

However, Fick's equation is applicable only at some minimal distance $\lambda$ from the solid surface, in most cases termed as the mean free path. The molecules escaping from the solid surface move in this range as free flyers rather than as randomly diffusing walkers. According to the limitations of Fick's theory, the lower limit of integration and the maximal concentration of the sample molecules have to be considered as determined at distance $\lambda$ from solid. Hence, eq 8 has to be rewritten in the form

$$
C(r)=C_{\max } \frac{r_{0}+\lambda}{r}
$$

A second issue that is important for small particles is that the division of eq 10 by the particle surface area, $2 \pi r_{0}^{2}$, to obtain the diffusion flow from a unit area, diverges as the particle size decreases. This leads to a paradox that the rate of sublimation can exceed the respective rate in vacuum. This inconsistency can be resolved if one takes into account the decrease in the maximal concentration of sample molecules near the solid surface as the particle size decreases. It is easy to obtain, using simple molecular kinetic theory together with the requirement for material conservation on a hemispherical boundary of radius $\left(r_{0}+\lambda\right)$, the variation of the maximal concentration $C_{\max }$ as a function of particle size. 


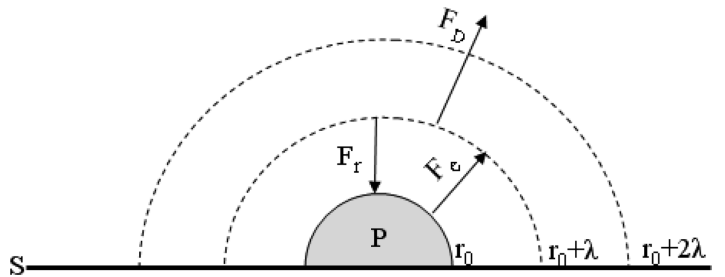

Figure 7. Scheme of molecular flows near the particle boundary and at a surface that is located at a distance $\lambda$ from the particle $\mathrm{P}$ surface. The fluxes: $F_{\mathrm{e}}, F_{\mathrm{r}}$ and $F_{\mathrm{D}}$ are flows associated with molecular desorption from the solid, readsorption of molecules to the solid, and molecular diffusion away from the solid, respectively.

Let us consider the molecular flows at the boundary $\left(r_{0}+\lambda\right)$ of a hemispherical particle as they shown in Figure 7. Three fluxes are considered: $F_{\mathrm{e}}, F_{\mathrm{r}}$, and $F_{\mathrm{D}}$. These represent the desorption of molecules from solid, the readsorption of gas TNT molecules by the solid, and the diffusion of TNT molecules away from the particle, respectively. These three fluxes have to be balanced. In the following we assume that sample molecule adsorption onto the substrate surface $\mathrm{S}$ can be neglected. It should be noted that including the adsorption of sample molecules onto the substrate would require considering of an additional process of surface diffusion. Since no effects associated with migration of TNT molecules on the substrate surface were observed, this process is neglected. Despite of reflection of the sample molecules from the substrate surface $S$, the spherical concentration distribution of the gas phase TNT molecules is conserved. Each of the three molecular flows in the Figure 7 can be found from molecular kinetic theory and Fick's equation, that is,

$$
\begin{gathered}
F_{\mathrm{e}}=\frac{A_{0} P_{\mathrm{sat}} v}{4 k_{\mathrm{B}} T}=\frac{1}{2} \frac{\pi r_{0}^{2} P_{\mathrm{sat}} v}{k_{\mathrm{B}} T} \\
F_{\mathrm{r}}=\frac{A_{0} C_{\text {max }} v}{4}=\frac{1}{2} \pi r_{0}^{2} C \max v \\
F_{\mathrm{D}}=-\mathrm{d} m / \mathrm{d} t=2 \pi D C_{\text {max }}\left(r_{0}+\lambda\right)
\end{gathered}
$$

where $v$ corresponds to the average thermal velocity that limits the sublimation rate in vacuum and is given by:

$$
v=\sqrt{\frac{8 k_{\mathrm{B}} T}{\pi M}}
$$

Balancing the flows $15-17$ leads to

$$
C_{\max }=\frac{P_{\text {sat }}}{k_{\mathrm{B}} T}\left[\frac{1}{1+\frac{4 D\left(r_{0}+\lambda\right)}{r_{0}{ }^{2} v}}\right]
$$

Substitution of eq 19 into 17 leads to the following expression for the sublimation rate, that replaces eq 10

$$
-\frac{\mathrm{d} m}{\mathrm{~d} t}=\frac{2 \pi D P_{\text {sat }}}{k_{\mathrm{B}} T} \frac{1}{\left(\frac{1}{r_{0}+\lambda}+\frac{4 \lambda}{3 r_{0}{ }^{2}}\right)}
$$

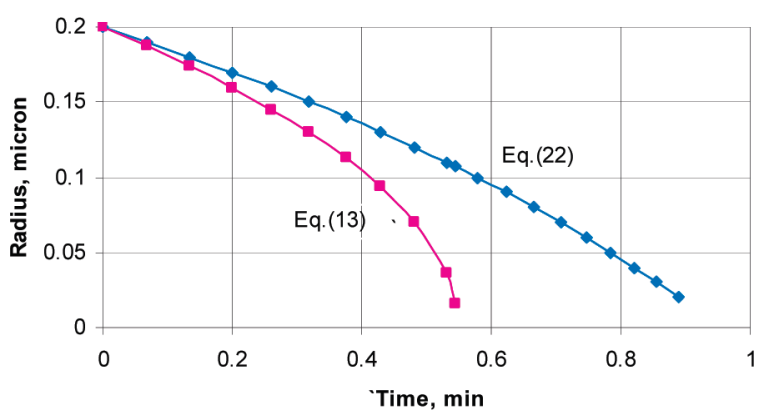

Figure 8. Time dependence of the size variation of a $0.2 \mu \mathrm{m}$ radius hemispherical TNT particle at $30{ }^{\circ} \mathrm{C}$.

with the mean free path $\lambda$ in air that is related to the diffusion coefficient by

$$
\lambda=\frac{3 D}{v}
$$

To obtain the particle size variation during sublimation one can integrate eq 20 in a similar manner to the one described above for larger particles, eq 10, to obtain the relation

$$
r_{0}^{2}-r(t)^{2}+\frac{2}{3} \lambda\left[r_{0}-r(t)\right]+2 \lambda^{2} \ln \left[\frac{r_{0}+\lambda}{r(t)+\lambda}\right]=\frac{2 D P_{\mathrm{sat}} M}{\rho k_{\mathrm{B}} T} t
$$

It should be noted that in integrating eq 20 one could take into account the dependence of the saturated vapor pressure on the particle size or on the interaction with a substrate, if required.

One can see that at $\lambda \ll r(t)$ the resultant eq 22 converts into eq 13 .

Figure 8 presents results of numerical calculations according to eq 22 for a hemispherical TNT particle with an initial radius of $0.2 \mu \mathrm{m}$ at $30^{\circ} \mathrm{C}$. The result that would be obtained using eq 13 is also shown for comparison. The difference in the asymptotic behavior of the two curves is clearly seen. It is obvious that eq 22 leads to a considerably slower sublimation of small (sub-micrometer) particles.

\section{Conclusion}

We have shown in this study that QCM experiments can be used for the measurement of sublimation rates of nonuniform layers of TNT microcrystals. The TNT particles were approximated as hemispherical microcrystals based on their measured average diameter and height. The approximation of the TNT particle by a hemispherical shape was crucial for the development of simple analytical expressions. It was demonstrated that sublimation rate of small TNT particles is controlled by molecular diffusion of the TNT vapor in the surrounding air. Comparison with data from the literature related to the sublimation of uniform TNT disk-like layers confirms this result. Following this conclusion, we obtained simple expressions to describe the particles size evolution during the sublimation process. The expression obtained to describe the sublimation rate allows the evaluation of the disappearance rate of TNT particles with the appropriate behavior for a wide size range, starting at very small sub-micrometer particles.

It should be noted that the results of the present study clearly indicates that traces of TNT particles are expected to evaporate after a few days. The actual lifetime of such particles depends 
on the conditions they are exposed to. This lifetime can be reliably estimated using the expressions developed here.

Acknowledgment. This work was partially supported by The Center of Excellence for Explosives Detection, Mitigation and Response, a Department of Homeland Security Center of Excellence in The University of Rhode Island.

\section{References and Notes} 403.

(1) Lenchitz, C.; Velicky, R. W. J. Chem. Eng. Data 1970, 15, 401-

(2) Pella, P. A. J. Chem. Thermodynamics 1977, 9, 301-305.

(3) Cundall, R. B.; Palmer, T. F.; Wood, C. E. C. J. Chem. Soc. Faraday Trans. 1978, 74, 1339-1345.

(4) Oxley, J. C.; Smith, J. L.; Shinde, K.; Moran, J. Propellants, Explosives, Pyrotechnics 2005, 30, 127-130.

(5) Edwards, G. Trans. Faraday Soc. 1950, 46, 423-427.

(6) Leggett, D. C. J. Chrom. 1977, 133, 83-90.

(7) McKone, T. E.; Daniels, J. I. Regul. Toxicol. Pharmacol. 1991, $13,36-61$.

(8) Phelan, J. M.; Webb, S. W. Environmental Fate and Transport of Chemical Signatures from Buried Landmines-Screening Model Formulation and Initial Simulations, Sandia Report SAND97-1426.US-741; Sandia National Laboratories: Albuquerque, Ca., 1997.
(9) Rosenblatt, D. H.; Burrows, E. P.; Mitchell, W. R.; Parmer, D. L. In The Handbook of Environmental Chemistry; Hutzinger, O., Ed.; SpringerVerlag: Berlin, 1989; pp 195-234.

(10) Dortch, M.; Zakikhani, M.; Furey, J; Meyer, R.; Fant, S.; Gerald, J.; Qasim, M; Fredrickson, H; Honea, P.; Bausum, H; Walker, K; Johnson, M. Data Gap Analysis and Database Expansion of Parameters for Munitions Constituents, Report ERDC/EL TR-05-16; US Army Corps of Engineers: WA, 2005; p 32.

(11) Mu, R.; Ueda, A.; Liu, Y. C.; Wu, M.; Henderson, D. O.; Lareau, R. T.; Chamberlain, R. T. Surface Sci. Lett. 2003, 530, L293-L296.

(12) Sauerbrey, G. Z. Phys. 1959, 155, 206-222

(13) Lu, C. S.; Lewis, O. J. Appl. Phys. 1972, 43, 4385-4390.

(14) Chao, Z.; Guanping, F. IEEE Trans. Ultrason., Ferroelectr., Frequency Control 1996, 43, 5.

(15) Bandey, H. L.; Martin, S. J.; Cernosek, R. W.; Hillman, A. R. Anal. Chem. 1999, 71, 2205-2214.

(16) Langmuir, I. Phys. Rev. Ser. II 1913, 2, 329.

(17) Knudsen, M. The Kinetic Theory of Gases: Methuen: London, 1950.

(18) Langmuir, I. Phys. Rev. 1918, 12, 368-370.

(19) Crank, J. Mathematics of Diffusion: Oxford University Press: Oxford, 1975,

(20) Gray, A.; Mathews, B. B.; MacRobert, T. M. A Treatise on Bessel Functions and their Applications to Physics: Macmillan and Co.Ltd: London, 1931.

(21) Mu, R.; Ueda, A.; Wu, M. H.; Tung, Y. S.; Henderson, D. O.; Chamberlain, R. T.; Curby, W.; Mercado, A. J. Phys. Chem. B 2000, 104 (1), 105-109.

JP105168H 